\title{
Who should be treated with pembrolizumab in addition to standard of care in advanced cervical cancer?
}

\author{
Seda Kahraman ${ }^{1}$ (1) $\cdot$ Ulviye Esra Erbas ${ }^{2} \cdot$ Bulent Yalcin $^{1}$ \\ Received: 18 November 2021 / Accepted: 28 November 2021 / Published online: 20 January 2022 \\ (c) The Author(s), under exclusive licence to Springer Science+Business Media, LLC, part of Springer Nature 2021
}

\begin{abstract}
Keynote-826 study demonstrated that the addition of pembrolizumab to standard systemic therapy contributes to progressionfree and overall survival in patients with persistent, recurrent, or metastatic cervical cancer receiving chemotherapy with or without bevacizumab. However, we do have some comments about the study. The lack of additional survival benefit of pembrolizumab in patients with de novo metastatic disease or in elderly patients or in patients with combined positive score (CPS) $<1$ may be due to the absence of abscopal immune effects of radiotherapy or primary resistance (immunodeficiency or immune escape or immunosenescence) to adaptive immunotherapy. We believe that new studies are needed to add pembrolizumab particularly in the treatment of the patients with de novo metastatic disease, PD-L1 CPS of $<1 \%$, and advanced cervical cancer in geriatric population.
\end{abstract}

Keywords Pembrolizumab $\cdot$ Advanced cervical cancer $\cdot$ First line therapy

To the Editor,

We read the article of Keynote-826 researchers with interest [1]. They demonstrated that the addition of pembrolizumab to standard systemic therapy contributes to progression-free and overall survival in patients with persistent, recurrent, or metastatic cervical cancer receiving chemotherapy with or without bevacizumab. However, we do have some comments about the study. In the subgroup analysis of the study, adding pembrolizumab to standard systemic therapy appears to be more effective in younger patients ( $<65$ years), patients with PD-L1 combined positive score (CPS) of $\geq 1$ and previously treated with regional interventions.

When the results of Keynote-826 study is evaluated, it is understood that adding pembrolizumab to standard first line therapy is not beneficial in almost more than $30 \%$ of the patients. The lack of additional survival benefit of pembrolizumab in patients with de novo metastatic disease or in elderly patients or in patients with CPS $<1$ may be due to the absence of abscopal

Seda Kahraman

sedakayacan.kahraman@gmail.com

1 Department of Medical Oncology, Ankara Yildirim Beyazit University, Ankara, Turkey

2 Faculty of Medicine, Ankara Yildirim Beyazit University, Ankara, Turkey immune effects of radiotherapy or primary resistance (immunodeficiency or immune escape) to adaptive immunotherapy. Additionally, how immune changes with aging, namely immunosenescence, relate to the incidence of cancer and the impact of immunotherapy in older adults is largely unknown.

We believe that the cost-effective use of immune checkpoint inhibitors in malignancies with proven benefits is crucial. In conclusion, new studies are needed to add pembrolizumab in the treatment of the patients with de novo metastatic disease, PD-L1 CPS of $<1 \%$, and advanced cervical cancer in geriatric population.

\section{Declarations}

Conflict of interest The author declares that there are no conflicts of interest.

\section{Reference}

1. Colombo N, et al. Pembrolizumab for Persistent, recurrent, or metastatic cervical cancer. N Engl J Med. 2021;385(20):1856-67.

Publisher's Note Springer Nature remains neutral with regard to jurisdictional claims in published maps and institutional affiliations. 